\title{
The effective non-linear properties of a composite coating and a composite sandwich layer ${ }^{\text {is }}$
}

\author{
N.A. Fleck ${ }^{\mathrm{a},}{ }^{*}$, J.R. Willis ${ }^{\mathrm{b}}$ \\ ${ }^{a}$ Cambridge University Engineering Dept., Trumpington St., Cambridge CB2 1PZ, UK \\ ${ }^{\mathrm{b}}$ Dept. Applied Mathematics and Theoretical Physics, Centre for Mathematical Sciences, Wilberforce Rd., Cambridge CB3 OWA, UK
}

\section{A R T I C L E I N F O}

\section{Article history:}

Received 2 February 2016

Accepted 11 February 2016

Available online 18 February 2016

\section{Keywords:}

Effective properties

Composites

Variational methods

\begin{abstract}
A B S T R A C T
Hashin-Shtrikman based bounds and estimates are obtained for the linear and non-linear effective properties of composites in the form of a thin coating or sandwich layer. It is assumed that the thickness of the layer is of the same order of magnitude as the correlation length between phases, and size effects thereby result. Boundary layers exist within the coating adjacent to the substrate and to the free surface (in the case of a coating). Attention is focused on two-dimensional problems by considering anti-plane shear of an isotropic 2-phase composite on a single-phase substrate, with microstructure prismatic along the direction of anti-plane shear.
\end{abstract}

() 2016 Published by Elsevier Masson SAS.

\section{Introduction}

Surface coatings and embedded layers are ubiquitous in engineering components, and serve a wide range of functions from environmental protection to low friction and wear resistance. Indeed, the field of surface engineering involves the manufacture of coatings with a wide range of multifunctional properties. The coating may be stiffer (and stronger) than that of the substrate, for example the surface layer of aluminium alloys can be converted to aluminium oxide by anodisation. Or, the coating may be softer and more compliant, such as zinc-coated steel, paints, low friction polymer coatings (such as PTFE on steel or aluminium alloy) and thermal barrier coatings. A related geometry to the surface coating is the embedded layer sandwiched between two substrates. This geometry is also ubiquitous and is representative of adhesive joints, the mortar between the bricks of a building, and interphases at grain boundaries inter alia.

Frequently, a coating comprises a multi-phase composite with, for example, particulate reinforcement in order to increase its stiffness and strength. The question arises: what are the effective properties of a composite coating? A common assumption is to use the effective properties of the bulk composite for that of the coating.

\footnotetext{
The paper is written to mark that N.A. Fleck received The Euromech Solid Mechanics Prize 2015 and that J.R. Willis received The Euromech Solid Mechanics Prize 2012.

* Corresponding author

E-mail address: naf1@eng.cam.ac.uk (N.A. Fleck).
}

Whilst this assumption is accurate when the correlation length of each phase is much less than the coating thickness, it is less accurate when the two length scales are of comparable magnitude. The presence of the substrate or a free surface perturbs the stress field within the composite coating. This can be re-phrased in a more mathematical manner, as follows. The usual Hashin-Shtrikman variational approach for the bulk properties of a composite makes use of the infinite-body Green's function in order to determine the ensemble-averaged strain field in terms of a polarization in stress from one phase to the next. For the embedded layer, the infinitebody Green's function is employed, whereas for a surface coating the half-space Greens function is exploited.

The purpose of this study is to make accurate predictions for the effective properties of a surface composite coating or an embedded composite layer, taking into account the presence of the substrate of differing properties, whether linear or non-linear. Effective properties and associated bounds are generated for composite coatings and for composite sandwich layers of finite thickness, based on the Hashin-Shtrikman approach, but suitably modified to account for the presence of a free surface in the case of a coating and of substrates in the case of a sandwich layer. First, the linear properties are generated and then the method is modified to generate bounds and estimates for a non-linear composite coating. We shall limit our scoping study to two-dimensional problems by considering antiplane shear of an isotropic 2-phase composite on a single-phase substrate, with microstructure prismatic along the direction of anti-plane shear. 


\section{Statement of problem: a composite half-space in antiplane shear: the 2D linear case}

We shall consider the anti-plane shear response of a coating of height $h$ made from a random $M$-phase composite, adhered to a monolithic substrate of height $H>>h$ made from phase $M+1$ material. The outer top surface of the coating is subjected to a longitudinal shear traction $\sigma_{y}^{\infty}$, while the base of the substrate is rigidly held without displacement, see Fig. 1a. Both the coating and substrate are initially treated as linear elastic, with the non-linear behaviour addressed in a subsequent section. The origin of a Cartesian reference frame $(x, y, z)$ is placed on the top external surface of the coating, with the $y$-direction aligned with the outward normal to the external surface. Thus, the coating extends over $-h \leq y \leq 0$, and the underlying substrate occupies $-(H+h) \leq y \leq-h$. The $z$ axis aligns with the direction of anti-plane shear. Results will be presented in the limit $H / h \rightarrow \infty$ but the recognition that $H$ is actually finite is needed to ensure convergence of certain integrals during the derivation.

A closely related problem is an $M$-phase composite layer of thickness $h$ sandwiched between two substrates made from phase $M+1$, with the assembled stack subjected to a longitudinal shear traction $\sigma_{y}^{\infty}$, see Fig. 1b. For this case, the origin of a Cartesian reference frame $(x, y, z)$ is placed on the upper interface of the coating, such that the coating extends over $-h \leq y \leq 0$, as shown in the figure. In our study, we shall focus on the coating problem of Fig. 1a but shall include the analysis and results for the sandwich layer at appropriate steps in the development.

The distribution of phases within the coating is taken to be isotropic, and each phase has a linear, isotropic response. In contrast, the isotropic substrate beneath the coating is taken to be homogeneous. We seek the effective properties of the coating. The single non-vanishing displacement $u(x, y)$ is in the $z$-direction. The resulting (engineering) shear strain has components $e_{x}=u,_{x}$ and $e_{y}=u, y$ and the work-conjugate stress has the shear components $\sigma_{x} \equiv \sigma_{z x}$ and $\sigma_{y} \equiv \sigma_{z y}$, respectively. For later convenience, a Greek suffix takes the values of $x$ or $y$, and a repeated Greek suffix denotes summation, in accordance with the usual Einstein notation. For example, $u_{, \alpha}$ denotes $u_{, x}$ or $u, y ; \sigma_{\alpha}$ denotes $\sigma_{x}$ or $\sigma_{y}$; and $u_{, \alpha \alpha}$ denotes $u_{, x x}+u_{, y y}$.
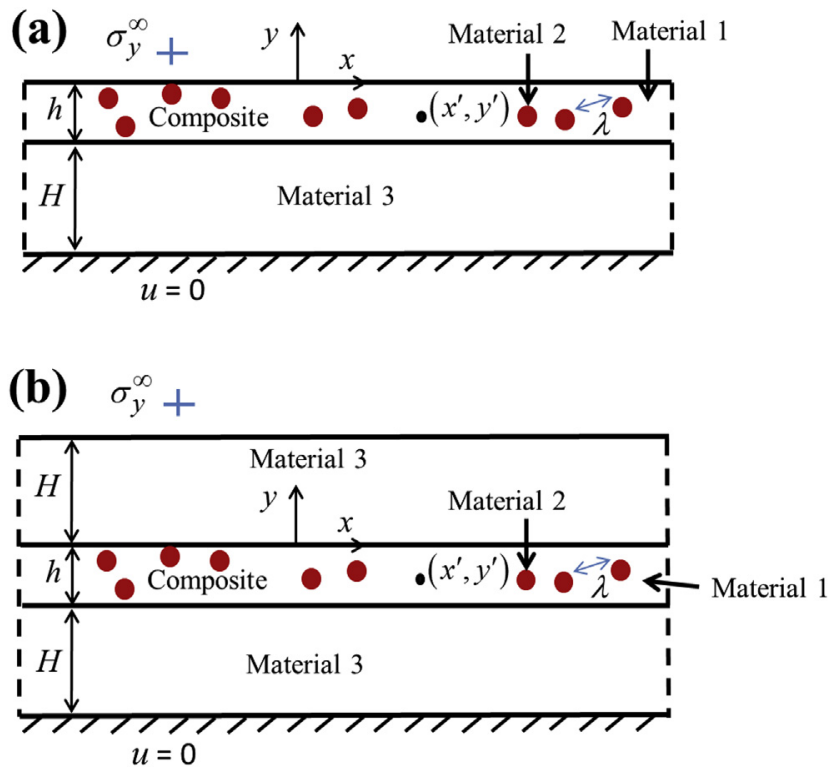

Fig. 1. (a) A coating and (b) a sandwich layer, comprising a two phase composite adhered to a substrate, under an imposed longitudinal shear traction $\sigma_{y}^{\infty}$.
The coating comprises an $M$-phase random composite, and each phase $r$ is isotropic and of shear modulus $\mu_{r}$. The substrate is homogeneous and isotropic, and is made from phase $M+1$ of shear modulus $\mu_{M+1}$. No variation in microstructure and material properties exists along the $z$-axis; recall that the applied surface shear traction $\sigma_{y}^{\infty}$ is also along this direction. The stress components $\sigma_{\alpha}$ at a given point $\boldsymbol{x}=(x, y)$ are related to the strain components at that point according to

$\sigma_{\alpha}=\mu e_{\alpha}=\mu u_{, \alpha}$

where $\mu(\boldsymbol{x})$ takes the value $\mu_{r}$ if $\boldsymbol{x}$ lies in material of type $r$. Thus,

$\mu(\boldsymbol{x})=\sum_{r=1}^{M+1} \mu_{r} \chi_{r}(\boldsymbol{x})$

where the characteristic function $\chi_{r}(\boldsymbol{x})$ takes the value of unity if material $r$ is at $\boldsymbol{x}$ and equals zero otherwise. We seek the overall effective response of the layer and substrate. First, we record the expressions for the bulk composite, as a benchmark.

\subsection{A summary of the effective response of an M-phase composite in shear}

It is instructive to compare the stiffness of the composite coating with that of the bulk composite. In order to do so, we assemble here the well-established results for the bulk response of a composite of volume fraction $p_{r}$ for each phase $r$. For completeness, we write the elementary bounds in the above notation. The elementary bounds imply a uniform strain distribution within the coating of magnitude $e_{y}=\sigma_{y}^{\infty} / \bar{\mu}$ where

$\bar{\mu}=\bar{\mu}_{V} \equiv \sum_{r=1}^{M} p_{r} \mu_{r}$

for the Voigt bound, and

$\bar{\mu}=\bar{\mu}_{R} \equiv\left(\sum_{r=1}^{M} p_{r} \mu_{r}^{-1}\right)^{-1}$

for the Reuss bound.

Hashin-Shtrikman bounds and estimates for the effective shear modulus of an $M$-phase composite have been derived by Hill (1964, 1965) and Walpole (1969). For a comparison medium of shear modulus $\mu_{0}$ the Hashin-Shtrikman estimate reads

$\mu_{\mathrm{HS}}=\left(\sum_{r=1}^{M} \frac{p_{r}}{\mu_{r}+\mu_{0}}\right)^{-1}-\mu_{0}$

The Hashin-Shtrikman upper bound $\mu_{\mathrm{HS}}^{+}$is attained by setting $\mu_{0}$ to the largest value of shear modulus over all phases. Likewise, the Hashin-Shtrikman lower bound $\mu_{\mathrm{HS}}^{-}$is attained by setting $\mu_{0}$ to the smallest value of shear modulus over all phases. And the selfconsistent estimate $\mu_{S C}$ is obtained by identifying $\mu_{0}$ with $\mu_{H S}$ in (2.5) and then solving for $\mu_{H S}$; a convenient way to accomplish this in the general case is to employ iteration to convergence of (2.5).

Now re-write (2.5) for the case of a 2-phase composite, and assume without loss of generality that $\alpha \equiv \mu_{2} / \mu_{1}>1$. Then,

$\frac{\mu_{\mathrm{HS}}^{+}}{\mu_{1}}=\frac{1+p_{1}+\alpha p_{2}}{1+p_{1}+\left(p_{2} / \alpha\right)}$ 
$\frac{\mu_{\mathrm{HS}}^{-}}{\mu_{1}}=\frac{p_{1}+\alpha\left(1+p_{2}\right)}{1+p_{2}+\alpha p_{1}}$.

In this case the self-consistent estimate takes the explicit form

$\frac{\mu_{\mathrm{SC}}}{\mu_{1}}=\frac{\left(p_{2}-p_{1}\right)(\alpha-1)}{2}+\left[\frac{\left(p_{2}-p_{1}\right)^{2}(\alpha-1)^{2}}{4}+\alpha\right]^{1 / 2}$

\section{The effective response of a linear $M$-phase composite coating and sandwich layer}

Now consider the problem of a linear $M$-phase composite, either in the form of a coating (of height $h$ ) on a substrate of phase $M+1$, or sandwiched between two substrates of phase $M+1$, recall Fig. 1 . The outer top surface of the coating is subjected to a longitudinal shear traction $\sigma_{y}^{\infty}$, see Fig. 1a or the sandwiched layer is subjected to the traction $\sigma_{y}^{\infty}$, see Fig. $1 \mathrm{~b}$. We shall introduce below the notion of a correlation length $\lambda$ between phases, and predict the effect of the presence of the free surface and substrate of the coating upon its effective properties: we shall calculate the effective shear modulus of the coating (and sandwich layer) as a function of the ratio $\lambda / h$. The Hashin-Shtrikman approach is adopted as follows.

Introduce a linear comparison material for the composite layer and the monolithic substrate(s), of shear modulus $\mu_{0}$, and re-write the constitutive relations (2.1) in the form

$\sigma_{\alpha}(\boldsymbol{x})=\mu_{0} e_{\alpha}(\boldsymbol{x})+\tau_{\alpha}(\boldsymbol{x})=\mu e_{\alpha}(\boldsymbol{x})$

in terms of a polarization field $\tau(\boldsymbol{x})$. Equilibrium dictates that the displacement field $u(x, y)$ satisfies

$\mu_{0} u_{, \alpha \alpha}+\tau_{\alpha, \alpha}=0$

Consider first the case of the coating. A solution to (3.2) is obtained in terms of a Green's function $G\left(\boldsymbol{x}, \boldsymbol{x}^{\prime}\right)$ for a point load at $\left(x^{\prime}, y^{\prime}\right)$ within a slab $-(H+h) \leq y \leq 0$ made from the linear comparison solid of shear modulus $\mu_{0}$. Define $G$ such that it satisfies

$\mu_{0} G_{, \alpha \alpha}+\delta\left(\boldsymbol{x}-\boldsymbol{x}^{\prime}\right)=0$

where $\delta$ is the usual 2D Dirac delta function. The solution of (3.3) can be expressed as

$G\left(\boldsymbol{x}, \boldsymbol{x}^{\prime}\right)=G^{\infty}\left(\boldsymbol{x}, \boldsymbol{x}^{\prime}\right)+G^{I}\left(\boldsymbol{x}, \boldsymbol{x}^{\prime}\right)$

where

$2 \pi \mu_{0} G^{\infty}\left(x, y ; x^{\prime}, y^{\prime}\right)=\ln \left(1 / r_{1}\right) ; \quad r_{1}^{2}=\left(x-x^{\prime}\right)^{2}+\left(y-y^{\prime}\right)^{2}$

The 'image' term $G^{I}$ is chosen so that $G$ satisfies the appropriate boundary conditions, namely that $G_{y}=0$ when $y=0$ and $G=0$ when $y=-(H+h)$, for all $y^{\prime} \in(-(H+h), 0)$. Its complete explicit form will not be needed but it is noted, for future use, that it reduces to

$2 \pi \mu_{0} G^{I}\left(x, y ; x^{\prime}, y^{\prime}\right) \sim \ln \left(1 / r_{2}\right)+c ; \quad r_{2}^{2}=\left(x-x^{\prime}\right)^{2}+\left(y+y^{\prime}\right)^{2}$

as $H / h \rightarrow \infty$, with $y$ and $y^{\prime}$ of order $h$ (and so $\boldsymbol{x}$ and $\boldsymbol{x}^{\prime}$ lie within or close to the surface layer). Here, $c$ is a constant that depends on $H$ but its exact value will not be needed in what follows. For the problem of the sandwich layer, $G^{I}$ is different but in the limit just mentioned it reduces to zero and thus may be disregarded entirely.
Multiply (3.2) by $G$, multiply (3.3) by $u$, and subtract one from the other to obtain

$$
\begin{gathered}
\mu_{0} u_{, \alpha \alpha} G-\mu_{0} G_{, \alpha \alpha} u \equiv\left(G \mu_{0} u_{, \alpha}\right)_{, \alpha}-\left(G_{, \alpha} \mu_{0} u\right)_{, \alpha} \\
=u(\boldsymbol{x}) \delta\left(\boldsymbol{x}-\boldsymbol{x}^{\prime}\right)-G\left(\boldsymbol{x}, \boldsymbol{x}^{\prime}\right) \tau_{\alpha, \alpha}(\boldsymbol{x})
\end{gathered}
$$

Now integrate (3.7) over the domain $\Omega$ occupied by the coating and underlying substrate, and integrate by parts to obtain

$u\left(\boldsymbol{x}^{\prime}\right)=u_{0}\left(\boldsymbol{x}^{\prime}\right)-\int_{\Omega} d \boldsymbol{x}\left[\frac{\partial G\left(\boldsymbol{x}, \boldsymbol{x}^{\prime}\right)}{\partial x_{\alpha}} \tau_{\alpha}(\boldsymbol{x})\right]$

where

$u_{0}\left(\boldsymbol{x}^{\prime}\right)=\int_{\partial \Omega} d S(\boldsymbol{x})\left[G\left(\mu_{0} u_{, \alpha}+\tau_{\alpha}\right) n_{\alpha}-u \mu_{0} G_{, \alpha} n_{\alpha}\right]$.

Note that the boundary conditions imposed for $G$ ensure that the integral in (3.8) involves only the prescribed boundary data, and $u_{0}$ is the solution of the given boundary value problem, for the 'comparison' body with shear modulus $\mu_{0}$. For the present simple boundary conditions, it is known directly that $u_{0}\left(\boldsymbol{x}^{\prime}\right)=\left(y^{\prime}+H+h\right) \sigma_{y}^{\infty} / \mu_{0}$.

The engineering shear strain follows immediately as

$e_{\beta}\left(\boldsymbol{x}^{\prime}\right)=\frac{\partial u\left(\boldsymbol{x}^{\prime}\right)}{\partial x_{\beta}^{\prime}}=\frac{\partial u_{0}\left(\boldsymbol{x}^{\prime}\right)}{\partial x_{\beta}^{\prime}}-\int_{\Omega} d \boldsymbol{x}\left[\frac{\partial^{2} G\left(\boldsymbol{x}, \boldsymbol{x}^{\prime}\right)}{\partial x_{\alpha} \partial x_{\beta}^{\prime}} \tau_{\alpha}(\boldsymbol{x})\right]$

in which the singularity at $\boldsymbol{x}=\boldsymbol{x}^{\prime}$ in the last integral is interpreted in the sense of distributions. Upon introducing the generalized function

$\Gamma_{\alpha \beta}\left(\boldsymbol{x}, \boldsymbol{x}^{\prime}\right)=\frac{\partial^{2} G\left(\boldsymbol{x}, \boldsymbol{x}^{\prime}\right)}{\partial x_{\alpha} \partial x_{\beta}^{\prime}}$

(3.10) can be formally re-expressed as

$e_{\beta}\left(\boldsymbol{x}^{\prime}\right)=e_{\beta}^{0}-\int_{\Omega} d \boldsymbol{x}\left[\Gamma_{\alpha \beta}\left(\boldsymbol{x}, \boldsymbol{x}^{\prime}\right) \tau_{\alpha}(\boldsymbol{x})\right]$,

where $e_{x}^{0}=0$ and $e_{y}^{0}=\sigma_{y}^{\infty} / \mu_{0}$.

3.1. The statistics of the composite and the Hashin-Shtrikman approximation for the polarization field

The composite is taken to be a random medium, such that $\chi_{r}(\boldsymbol{x})$ is a random field. The probability that material $r$ is at $\boldsymbol{x}$ is given by the ensemble average of $\chi_{r}(\boldsymbol{x})$, written as

$p_{r}(\boldsymbol{x})=\left\langle\chi_{r}(\boldsymbol{x})\right\rangle$

Similarly, the probability of finding simultaneously material of type $r$ at $\boldsymbol{x}$ and type $s$ at $\boldsymbol{x}^{\prime}$ is

$p_{r s}\left(\boldsymbol{x}, \boldsymbol{x}^{\prime}\right)=\left\langle\chi_{r}(\boldsymbol{x}) \chi_{s}\left(\boldsymbol{x}^{\prime}\right)\right\rangle$

The substrate $(r=M+1)$ is included within this framework by taking $\chi_{M+1}(\boldsymbol{x}) \equiv 1$ if $\boldsymbol{x}$ is in the substrate and zero otherwise. Then, $p_{M+1}(\boldsymbol{x})=1$ in the substrate and zero in the coating, $p_{M+1 s}\left(\boldsymbol{x}, \boldsymbol{x}^{\prime}\right)=p_{M+1}(\boldsymbol{x}) p_{s}\left(\boldsymbol{x}^{\prime}\right)$ and $p_{r M+1}\left(\boldsymbol{x}, \boldsymbol{x}^{\prime}\right)=p_{r}(\boldsymbol{x}) p_{M+1}\left(\boldsymbol{x}^{\prime}\right)$.

We shall take the composite material from which the coating is made to be statistically uniform (i.e. the statistics are those of a stationary random process), such that $p_{r}(\boldsymbol{x})$ is independent of the position $\boldsymbol{x}$, and equals the volume fraction $p_{r}$ of phase $r$; likewise, 
$p_{r s}\left(\boldsymbol{x}, \boldsymbol{x}^{\prime}\right)$ depends only upon the relative position $\left(\boldsymbol{x}-\boldsymbol{x}^{\prime}\right)$ and can be rewritten as $p_{r s}\left(\boldsymbol{x}, \boldsymbol{x}^{\prime}\right)$. In this initial study we shall consider a statistically isotropic composite, such that the $p_{r s}\left(\boldsymbol{x}, \boldsymbol{x}^{\prime}\right)$ depends only upon the radial separation $\left|\boldsymbol{x}-\boldsymbol{x}^{\prime}\right|$, with the property that $p_{r s}(\boldsymbol{x}) \rightarrow p_{r} p_{s}$ as $|\boldsymbol{x}| \rightarrow \infty$ assuming that no long-range order exists.

Symmetry dictates that $\tau_{x}^{(r)}=0$, and so it only remains to solve for $\tau_{y}^{(r)}$. Now make the Hashin-Shtrikman type approximation that the polarization $\tau_{y}$ has the value $\tau_{y}^{(r)}(y)$ within each phase $r$, so that we may write

$\tau_{y}(\boldsymbol{x})=\sum_{r=1}^{M+1} \tau_{y}^{(r)}(y) \chi_{r}(\boldsymbol{x})$

and proceed to solve for $\tau_{y}^{(r)}(y)$. Upon making use of (2.1), the relation (3.1) can be re-expressed as

$e_{y}(\boldsymbol{x})=\left(\mu-\mu_{0}\right)^{-1} \tau_{y}(\boldsymbol{x})$

Substitution of (2.1) into (3.12) provides for phase $r$,

$$
\begin{aligned}
& \left(\mu_{r}-\mu_{0}\right)^{-1} \tau_{y}^{(r)}\left(y^{\prime}\right) \chi_{r}\left(\boldsymbol{x}^{\prime}\right)+\int_{\Omega} d \boldsymbol{x}\left[\Gamma_{y y}\left(\boldsymbol{x}, \boldsymbol{x}^{\prime}\right) \sum_{s=1}^{M+1} \tau_{y}^{(s)}(y) \chi_{s}(\boldsymbol{x}) \chi_{r}\left(\boldsymbol{x}^{\prime}\right)\right] \\
& \quad=e_{y}^{0}\left(\boldsymbol{x}^{\prime}\right) \chi_{r}\left(\boldsymbol{x}^{\prime}\right) .
\end{aligned}
$$

This system is over-determined and has no exact solution. An approximation which takes into account two-point statistics is obtained by ensemble averaging, to give

$$
\begin{aligned}
& p_{r}\left(\mu_{r}-\mu_{0}\right)^{-1} \tau_{y}^{(r)}\left(y^{\prime}\right)+\int_{\Omega} d \boldsymbol{x}\left[\Gamma_{y y}\left(\boldsymbol{x}, \boldsymbol{x}^{\prime}\right) \sum_{s=1}^{M+1} p_{r s}\left(\boldsymbol{x}, \boldsymbol{x}^{\prime}\right) \tau_{y}^{(s)}(y)\right] \\
& \quad=e_{y}^{0}\left(\boldsymbol{x}^{\prime}\right) p_{r}
\end{aligned}
$$

An alternative derivation of this equation, based on the minimum energy principle, is given in Section 5.

It remains to solve for the polarization $\tau_{y}^{(r)}$ in each phase. Some further reduction of (3.18) is possible, by noting that the two point correlation function $p_{r s}\left(\boldsymbol{x}, \boldsymbol{x}^{\prime}\right)$ tends to the limit $p_{r} p_{s}$ as $\left|\boldsymbol{x}-\boldsymbol{x}^{\prime}\right| \rightarrow \infty$ and tends to the value $\delta_{r s} p_{r}$ (no sum on $r$ ) as $\left|\boldsymbol{x}-\boldsymbol{x}^{\prime}\right| \rightarrow 0$. Accordingly, we re-write $p_{r s}\left(\boldsymbol{x}, \boldsymbol{x}^{\prime}\right)$ as

$p_{r s}\left(\boldsymbol{x}, \boldsymbol{x}^{\prime}\right)=\psi_{r s}\left(\boldsymbol{x}, \boldsymbol{x}^{\prime}\right)+p_{r} p_{s}$

and re-express (3.18) as

$p_{r}\left(\mu_{r}-\mu_{0}\right)^{-1} \tau_{y}^{(r)}\left(\boldsymbol{x}^{\prime}\right)+I_{1}^{(r)}\left(\boldsymbol{x}^{\prime}\right)+I_{2}^{(r)}\left(\boldsymbol{x}^{\prime}\right)=e_{y}^{0} p_{r}$

where

$I_{1}^{(r)}\left(\boldsymbol{x}^{\prime}\right)=\int_{\Omega} d \boldsymbol{x}\left[\Gamma_{y y}\left(\boldsymbol{x}, \boldsymbol{x}^{\prime}\right) \sum_{s=1}^{M+1} p_{r} p_{s} \tau_{y}^{(s)}(y)\right]$

and

$I_{2}^{(r)}\left(\boldsymbol{x}^{\prime}\right)=\int_{\Omega} d \boldsymbol{x}\left[\Gamma_{y y}\left(\boldsymbol{x}, \boldsymbol{x}^{\prime}\right) \sum_{s=1}^{M} \psi_{r s}\left(\boldsymbol{x}, \boldsymbol{x}^{\prime}\right) \tau_{y}^{(s)}(y)\right]$

This last sum extends only to $M$ because $\psi_{r M+1}=\psi_{M+1 s}=0$.

Since $\tau_{y}^{(s)}$ is a function only of the co-ordinate $y$, (3.21) can be evaluated directly to give
$I_{1}^{(r)}\left(\boldsymbol{x}^{\prime}\right)=\frac{1}{\mu_{0}} \sum_{s=1}^{M+1} p_{r} p_{s} \tau_{y}^{(s)}\left(y^{\prime}\right)$

by the following argument. Note first that $G^{\infty}$ depends on $\boldsymbol{x}, \boldsymbol{x}^{\prime}$ only in the combination $\boldsymbol{x}-\boldsymbol{x}^{\prime}$ and by itself satisfies (3.3). The image term $G^{I}$ has a more complex structure but $G=G^{\infty}+G^{I}$ depends on $x, x^{\prime}$ only in the combination $x-x^{\prime}$, and its Fourier transform

$\tilde{G}\left(\xi, y ; y^{\prime}\right)=\int_{-\infty}^{\infty} e^{i \xi x} G\left(x, y ; y^{\prime}\right) d x$

is readily found to be

$$
\begin{aligned}
\tilde{G}\left(\xi, y ; y^{\prime}\right)= & \frac{e^{-|\xi|\left|y-y^{\prime}\right|}}{2 \mu_{0}|\xi|}+\frac{e^{|\xi|\left(y+y^{\prime}\right)}}{2 \mu_{0}|\xi|} \tanh |\xi|(H+h) \\
& +\frac{e^{|\xi|\left(y^{\prime}-y\right)}}{2 \mu_{0}|\xi|}[\tanh |\xi|(H+h)-1] .
\end{aligned}
$$

Hence,

$$
\begin{aligned}
\tilde{\Gamma}_{y y}\left(\xi, y ; y^{\prime}\right)= & \frac{1}{\mu_{0}} \delta\left(y-y^{\prime}\right)-\frac{|\xi| e^{-|\xi|\left|y-y^{\prime}\right|}}{2 \mu_{0}} \\
& +\frac{|\xi| e^{|\xi|\left(y+y^{\prime}\right)}}{2 \mu_{0}} \tanh |\xi|(H+h) \\
& -\frac{|\xi| e^{|\xi|\left(y^{\prime}-y\right)}}{2 \mu_{0}}[\tanh |\xi|(H+h)-1] .
\end{aligned}
$$

The result

$$
\int_{-\infty}^{\infty} \Gamma_{y y} d x=\frac{1}{\mu_{0}} \delta\left(y-y^{\prime}\right)
$$

follows immediately by setting $\xi=0$. Consequently,

$\int_{-(H+h)}^{0} d y^{\prime} \int_{-\infty}^{\infty} d x^{\prime}\left[\frac{\partial^{2} G\left(\boldsymbol{x}, \boldsymbol{x}^{\prime}\right)}{\partial y \partial y^{\prime}} \tau_{y}^{(s)}\left(y^{\prime}\right)\right]=\frac{1}{\mu_{0}} \tau_{y}^{(s)}(y)$

and (3.23) follows immediately. For the substrate, we take $r=M+1$ and Equation (3.20) gives

$\left[\left(\mu_{M+1}-\mu_{0}\right)^{-1}+\left(\mu_{0}\right)^{-1}\right] \tau_{y}^{(M+1)}(y)=e_{y}^{0} \equiv \sigma_{y}^{\infty} / \mu_{0}$.

It is easily checked that this delivers the exact strain $e=\sigma_{y}^{\infty} / \mu_{M+1}$ in the substrate, regardless of the choice of $\mu_{0}$.

For the coating, some further reduction of (3.22) is needed. Since both $\boldsymbol{x}$ and $\boldsymbol{x}^{\prime}$ are confined to the coating, the asymptotic approximation (3.6) for $G^{I}$ is employed. The integrand of (3.22) is singular and must be interpreted in the sense of generalized functions. Expand $\psi_{r s}\left(\boldsymbol{x}, \boldsymbol{x}^{\prime}\right)$ about the point $\boldsymbol{x}=\boldsymbol{x}^{\prime}$ such that

$\psi_{r s}\left(\boldsymbol{x}, \boldsymbol{x}^{\prime}\right)=\psi_{r s}\left(\boldsymbol{x}^{\prime}, \boldsymbol{x}^{\prime}\right)+\left(\psi_{r s}\left(\boldsymbol{x}, \boldsymbol{x}^{\prime}\right)-\psi_{r s}\left(\boldsymbol{x}^{\prime}, \boldsymbol{x}^{\prime}\right)\right)$

and note that

$\psi_{r s}\left(\boldsymbol{x}^{\prime}, \boldsymbol{x}^{\prime}\right)=p_{r}\left(\delta_{r s}-p_{s}\right) \quad($ no sum on $\mathrm{r})$

For numerical convenience, we partition the domain of integration in (3.22) into a small square $\mathrm{D}$, of side length $d$ and centred on $\boldsymbol{x}=\boldsymbol{x}^{\prime}$, and a second domain $(\Omega-\mathrm{D})$ comprising the surface coating and substrate, but external to the square domain centred on 
$\boldsymbol{x}=\boldsymbol{x}^{\prime}$. Then, (3.22) reads

$I_{2}^{(r)}\left(\boldsymbol{x}^{\prime}\right)=I_{\mathrm{D}}^{(r)}\left(\boldsymbol{x}^{\prime}\right)+I_{\Omega-\mathrm{D}}^{(r)}\left(\boldsymbol{x}^{\prime}\right)$

where

$I_{\mathrm{D}}^{(r)}\left(\boldsymbol{x}^{\prime}\right)=\int_{\mathrm{D}} d \boldsymbol{x}\left[\left(\frac{\partial^{2} G^{\infty}\left(\boldsymbol{x}, \boldsymbol{x}^{\prime}\right)}{\partial y \partial y^{\prime}}+\frac{\partial^{2} G^{\mathrm{I}}\left(\boldsymbol{x}, \boldsymbol{x}^{\prime}\right)}{\partial y \partial y^{\prime}}\right) \sum_{s=1}^{M} \psi_{r s}\left(\boldsymbol{x}, \boldsymbol{x}^{\prime}\right) \tau_{y}^{(s)}(\boldsymbol{x})\right]$

and

$$
\begin{aligned}
I_{\Omega-\mathrm{D}}^{(r)}\left(\boldsymbol{x}^{\prime}\right)= & \int_{\Omega-\mathrm{D}} d \boldsymbol{x}\left[\left(\frac{\partial^{2} G^{\infty}\left(\boldsymbol{x}, \boldsymbol{x}^{\prime}\right)}{\partial y \partial y^{\prime}}+\frac{\partial^{2} G^{\mathrm{I}}\left(\boldsymbol{x}, \boldsymbol{x}^{\prime}\right)}{\partial y \partial y^{\prime}}\right)\right. \\
& \left.\times \sum_{S=1}^{M} \psi_{r s}\left(\boldsymbol{x}, \boldsymbol{x}^{\prime}\right) \tau_{y}^{(s)}(\boldsymbol{x})\right]
\end{aligned}
$$

Provided the square domain is sufficiently small (we shall take $d / h=10^{-3}$ in subsequent numerical simulations) the integral (3.33) over $\mathrm{D}$ is dominated by the contribution from the singularity in $\frac{\partial^{2} G^{\infty}\left(\boldsymbol{x}, \boldsymbol{x}^{\prime}\right)}{\partial y \partial y^{\prime}}$ at $\boldsymbol{x}=\boldsymbol{x}^{\prime}$. In particular, note that

$\int_{D} d \boldsymbol{x}\left[\frac{\partial^{2} G^{\infty}\left(\boldsymbol{x}, \boldsymbol{x}^{\prime}\right)}{\partial y \partial y^{\prime}}\right]=\frac{1}{2 \mu_{0}}$

by the following argument. Recall from (3.5(i)) that $G^{\infty}\left(\boldsymbol{x}, \boldsymbol{x}^{\prime}\right)$ is radially symmetric about $\boldsymbol{x}=\boldsymbol{x}^{\prime}$ Consequently,

$\int_{\mathrm{D}} d \boldsymbol{x}^{\prime}\left[\frac{\partial^{2} G^{\infty}\left(\boldsymbol{x}, \boldsymbol{x}^{\prime}\right)}{\partial y^{\prime} \partial y^{\prime}}\right]=\int_{\mathrm{D}} d \boldsymbol{x}^{\prime}\left[\frac{\partial^{2} G^{\infty}\left(\boldsymbol{x}, \boldsymbol{x}^{\prime}\right)}{\partial x^{\prime} \partial x^{\prime}}\right]$

and integration of (3.3) over D provides

$\int_{D} d \boldsymbol{x}^{\prime}\left[\frac{\partial^{2} G^{\infty}\left(\boldsymbol{x}, \boldsymbol{x}^{\prime}\right)}{\partial y^{\prime} \partial y^{\prime}}\right]=-\frac{1}{2 \mu_{0}}$

The result (3.35) follows immediately. Upon making use of (3.31) the expression for (3.33) simplifies to

$I_{\mathrm{D}}^{(r)}\left(\boldsymbol{x}^{\prime}\right)=\frac{1}{2 \mu_{0}} \sum_{s=1}^{M} p_{r}\left(\delta_{r s}-p_{s}\right) \tau_{y}^{(s)}\left(\boldsymbol{x}^{\prime}\right) \quad($ no sum on $\mathrm{r})$

Now substitute (3.23), (3.34) and (3.38) into (3.20), to obtain the governing integral equation

$$
\begin{aligned}
& p_{r}\left(\mu_{r}-\mu_{0}\right)^{-1} \tau_{y}^{(r)}\left(\boldsymbol{x}^{\prime}\right)+\frac{1}{2 \mu_{0}} \sum_{s=1}^{M}\left[p_{r}\left(\delta_{r s}+p_{s}\right) \tau_{y}^{(s)}\left(\boldsymbol{x}^{\prime}\right)\right] \\
& \quad+\int_{\Omega-D} d \boldsymbol{x}\left[\Gamma_{y y}\left(\boldsymbol{x}, \boldsymbol{x}^{\prime}\right) \sum_{s=1}^{M} \psi_{r s}\left(\boldsymbol{x}, \boldsymbol{x}^{\prime}\right) \tau_{y}^{(s)}(\boldsymbol{x})\right] \\
& \quad=e_{y}^{0}\left(\boldsymbol{x}^{\prime}\right) p_{r} \quad \text { (no sum on r) }
\end{aligned}
$$

for the polarization $\tau_{y}^{(r)}\left(\boldsymbol{x}^{\prime}\right)$ in each phase $r=1,2, \ldots, M$.

The ensemble average strain $\left\langle e_{y}\right\rangle\left(\boldsymbol{x}^{\prime}\right)$ in the coating can be determined from the polarization distribution $\tau_{y}^{(r)}\left(\boldsymbol{x}^{\prime}\right)$ as follows. Upon taking the ensemble average of (3.12) we obtain $\left\langle e_{y}\right\rangle\left(\boldsymbol{x}^{\prime}\right)=e_{y}^{0}\left(\boldsymbol{x}^{\prime}\right)-\int_{\Omega} d \boldsymbol{x}\left[\Gamma_{y y}\left(\boldsymbol{x}, \boldsymbol{x}^{\prime}\right) \sum_{s=1}^{M} p_{s} \tau_{y}^{(s)}(\boldsymbol{x})\right]$

Since $\tau_{y}^{(s)}$ is a function only of the co-ordinate $y$, (3.40) simplifies to

$\left\langle e_{y}\right\rangle(y)=e_{y}^{0}(y)-\frac{1}{\mu_{0}} \sum_{s=1}^{M} p_{s}(y) \tau_{y}^{(s)}(y)$

Note that, within the Hashin-Shtrikman approximation, the response of the coating does not depend upon the properties of the substrate.

\subsubsection{Additional assumption: the two phase composite}

At this stage in the calculation we specialise to the case of a two phase medium, $M=2$, with isotropic statistics in order to simplify the third term on the left-hand side of (3.39). First, note the connections

$$
\begin{aligned}
p_{11}(\boldsymbol{x})-p_{1} p_{1} & =-\left(p_{12}(\boldsymbol{x})-p_{1} p_{2}\right)=-\left(p_{21}(\boldsymbol{x})-p_{1} p_{2}\right) \\
& =p_{22}(\boldsymbol{x})-p_{2} p_{2}=p_{1} p_{2} h(r)
\end{aligned}
$$

where the correlation functions can be expressed by a specified radial function $h(r)$ since the statistics are taken to be isotropic. Substitution of these connections into (3.19) gives

$\psi_{11}=\psi_{22}=-\psi_{12}=-\psi_{21}=p_{1} p_{2} h(r)$

For simplicity, we shall follow the choice made by Smyshlyaev and Fleck (1995) and Drugan (2003), and take

$h(r)=e^{-r / \lambda}$

so that $\lambda$ is a correlation length scale for the microstructure. (Drugan showed that the exponential two-point correlation function gives excellent agreement with the result calculated from the Verlet-Weis improvement to the Percus-Yevick model in 3D, see Fig. 1 of Drugan (2003). We assume a similar functional form in the 2D case.)

\section{Hashin-Shtrikman bounds and self-consistent estimates}

There remains choice in the value of shear modulus for the comparison medium. First, take the case $\mu_{0}=\mu_{1}$, second $\mu_{0}=\mu_{2}$ and third, consider the self-consistent case.

(i) The choice $\mu_{0}=\mu_{1}$. The polarization $\tau_{y}^{(1)}\left(y^{\prime}\right)$ vanishes in phase 1 . Then, $\tau_{y}^{(2)}\left(y^{\prime}\right)$ within phase 2 of the coating is obtained by substituting (3.43) into (3.39), to give

$$
\begin{aligned}
& \frac{2+\left(1+p_{2}\right)(\alpha-1)}{2(\alpha-1)} \tau_{y}^{(2)}\left(\boldsymbol{x}^{\prime}\right)+\int_{\Omega_{C}-D} d \boldsymbol{x}\left[p_{1} \mu_{1} \Gamma_{y y}\left(\boldsymbol{x}, \boldsymbol{x}^{\prime}\right) h(r) \tau_{y}^{(2)}(\boldsymbol{x})\right] \\
& =\sigma_{y}^{\infty}
\end{aligned}
$$

where $\alpha \equiv \mu_{2} / \mu_{1}$ and the domain of integration $\Omega_{C}$ is restricted to that of the coating since the contribution from the substrate vanishes. The strain distribution within the coating follows immediately from (3.41) to give

$\left\langle e_{y}\right\rangle(y)=\frac{\sigma_{y}^{\infty}-p_{2} \tau_{y}^{(2)}(y)}{\mu_{1}}$

in addition to a uniform strain of $\left\langle e_{y}\right\rangle(y)=\sigma^{\infty} / \mu_{1}$ (and vanishing 
polarisation) within the substrate. Note that this solution corresponds to a Hashin-Shtrikman lower bound on energy for the case where phase 2 is stiffer than phase 1 . Similarly, the solution corresponds to a Hashin-Shtrikman upper bound on energy for the case where phase 2 is more compliant that phase 1 . The effective average shear modulus across the coating $\bar{\mu}$ is obtained from the average value of $\left\langle e_{y}\right\rangle(y)$ across the coating, such that

$$
\bar{\mu}=\frac{h \sigma_{y}^{\infty}}{\int_{-h}^{0}\left\langle e_{y}\right\rangle(y) d y}
$$

The integral Equation (4.1) is solved using standard MATLAB routines by sub-dividing the thickness of the coating into a large number $n$ of sub-layers, and by assuming that $\tau_{y}^{(2)}\left(y^{\prime}\right)$ is piecewise constant within each sub-layer. The operator $\Gamma_{y y}\left(\mathrm{x}^{\prime}, \mathrm{x}\right)$ is expressed in algebraic form by substituting (3.4) into (3.11), but the expression is omitted here for the sake of brevity.

(ii) The choice $\mu_{0}=\mu_{2}$. Then, the polarization vanishes in phase 2 of the coating. The polarization $\tau_{y}^{(1)}\left(y^{\prime}\right)$ within the coating is obtained by substituting (3.43) into (3.39), to give

$$
\begin{aligned}
& \frac{2 \alpha+\left(1+p_{1}\right)(1-\alpha)}{2(1-\alpha)} \tau_{y}^{(1)}\left(\boldsymbol{x}^{\prime}\right)+\int_{\Omega_{C}-D} d \boldsymbol{x}\left[p_{2} \mu_{2} \Gamma_{y y}\left(\boldsymbol{x}, \boldsymbol{x}^{\prime}\right) h(r) \tau_{y}^{(1)}(\boldsymbol{x})\right] \\
& \quad=\sigma_{y}^{\infty}
\end{aligned}
$$

where the domain of integration $\Omega_{C}$ is again restricted to that of the coating. The strain distribution within the coating follows immediately from (3.41) to give

$$
\left\langle e_{y}\right\rangle(y)=\frac{\sigma_{y}^{\infty}-p_{1} \tau_{y}^{(1)}(y)}{\mu_{2}}
$$

Note that this solution corresponds to a Hashin-Shtrikman lower bound on energy for the case where phase 1 is stiffer than phase 2 . Similarly, the solution corresponds to a Hashin-Shtrikman upper bound on energy for the case where phase 1 is more compliant that phase 2 . The effective average shear modulus across the coating $\bar{\mu}$ is again given by (4.3).

As discussed above for case (i), the integral Equation (4.4) is solved using standard MATLAB routines by sub-dividing the thickness of the coating into a large number $n$ of sub-layers, and by assuming that $\tau_{y}^{(1)}\left(y^{\prime}\right)$ is piecewise constant within each sub-layer. After $\tau_{y}^{(1)}\left(y^{\prime}\right)$ has been determined, the strain distribution $\left\langle e_{y}\right\rangle(y)$ within the coating is calculated via (4.5).

(iii) A self-consistent estimate is obtained by judicious choice of the shear modulus of the comparison solid $\mu_{0}$ in (3.39). We propose an iterative procedure, such that the average shear modulus over the height of the coating $\bar{\mu}$ is calculated for any assumed value of linear comparison solid, $\mu_{0}$. As an initial guess, we equate $\mu_{0}$ to the Hashin-Shtrikman self-consistent value for a bulk composite. Then, we solve for $\tau_{y}^{(r)}\left(y^{\prime}\right)$ within each phase $r=1,2$ of the coating by solving the pair of integral Equation (3.39). The ensemble average shear strain distribution within the coating is specified by (3.41). Next, take the average value of $\left\langle e_{y}\right\rangle\left(y^{\prime}\right)$ over the height $h$ of the coating and denote this as $\bar{e}_{y}$. Then, the average shear modulus is $\bar{\mu} \equiv \sigma_{y}^{\infty} / \bar{e}_{y}$ and we update our choice for $\mu_{0}$. In turn, $\tau_{y}^{(r)}\left(y^{\prime}\right),\left\langle e_{y}\right\rangle\left(y^{\prime}\right)$ and $\bar{\mu}$ are calculated for the updated value of $\mu_{0}$, and so on until a converged solution is obtained for $\mu_{0}$.

\section{Non-linear variational principle}

\subsection{Upper bound}

Consider a composite with an isotropic distribution of isotropic non-linear phases. Assume that each phase has a power-law response, such that the strain energy density for phase $r$ is given by

$w_{r}\left(e_{\alpha}\right)=\frac{\sigma_{r} e_{0}}{N+1}\left(\frac{e}{e_{0}}\right)^{N+1}$

in terms of a strength $\sigma_{r}$, hardening exponent $N<1$ and an effective strain

$e=\left(e_{\alpha} e_{\alpha}\right)^{1 / 2}$

We obtain an approximate solution in this case by developing a scheme that takes some ideas from the procedure of Ponte Castañeda $(1991,1992)$ for finding an upper-bound estimate for the nonlinear response of a statistically-uniform composite. This starts from a variational principle for a finite body, so we define the domain $V$ to be our layered structure, restricted to the range $-L<x<L$, with $L \gg H$. Zero tractions will be applied on the ends $x= \pm L$. The problem for this finite body can be formulated as the minimum energy principle

$\bar{U}=\inf _{u \in K} \frac{1}{V} \int_{V} d \boldsymbol{x}\left[w\left(e_{\alpha}, \boldsymbol{x}\right)-\sigma_{\beta}^{0} e_{\beta}\right]$

where

$w\left(e_{\alpha}, \boldsymbol{x}\right)=\sum_{r=1}^{M} w_{r}\left(e_{\alpha}\right) \chi_{r}(\boldsymbol{x})$

in the composite layer, and takes the form (5.1), with $r=M+1$ in the substrate. $K$ is the set of displacements that are zero on the base $y=-(H+h)$ of the substrate, and $\sigma_{\beta}^{0}$ is any stress field that satisfies the equilibrium equation and the traction boundary conditions; in the present context, we adopt $\sigma_{x}^{0}=0$ and $\sigma_{y}^{0}=\sigma_{y}^{\infty}$.

Now following Ponte Castañeda $(1991,1992)$, we introduce a comparison linear composite with the same microgeometry as the actual composite. This linear composite has energy density function

$W_{L}\left(e_{\alpha}, \boldsymbol{x}\right)=\frac{1}{2} \mu(\boldsymbol{x}) e^{2}$

where $\mu(\boldsymbol{x})$ takes the value $\mu_{r}$ in phase $r$. It follows immediately that

$\bar{U} \leq \inf _{u \in K} \frac{1}{V} \int_{V} d \boldsymbol{x}\left[w_{L}\left(e_{\alpha}, \boldsymbol{x}\right)+\max _{e_{\gamma}}\left\{w\left(e_{\gamma}, \boldsymbol{x}\right)-w_{L}\left(e_{\gamma}, \boldsymbol{x}\right)\right\}-\sigma_{\beta}^{0} e_{\beta}\right]$

for any choice of the parameters $\mu_{r}$. Explicitly, in phase $r$, the maximum in (5.6) is attained when

$\frac{e}{e_{0}}=\left(\frac{\sigma_{r}}{e_{0} \mu_{r}}\right)^{\frac{1}{1-N}}$

and routine manipulation gives

$\max _{e_{\gamma}}\left\{w\left(e_{\gamma}, \boldsymbol{x}\right)-w_{L}\left(e_{\gamma}, \boldsymbol{x}\right)\right\}=\frac{(1-N)}{2(1+N)} e_{0} \sigma_{r}\left(\frac{\sigma_{r}}{e_{0} \mu_{r}}\right)^{\frac{1+N}{1-N}}$ 
The infimum over $u$ in (5.6) cannot be calculated explicitly but it can be approximated using the Hashin-Shtrikman methodology already described, which employs a trial displacement field generated by the representation (3.9) relative to a comparison medium with shear modulus $\mu_{0}$ [except that $\Omega$ is replaced by the domain $V$ of width $2 L$ ] in conjunction with the approximation (3.15) for $\tau_{\alpha}$. Explicitly, in terms of $\tau_{\alpha}$,

$$
\begin{aligned}
\bar{U} \leq & \inf _{\tau_{\alpha}} \frac{1}{V} \int_{V} d \boldsymbol{x}^{\prime}\left[\left\{\frac{1}{2} \mu_{0}\left[e\left(\boldsymbol{x}^{\prime}\right)\right]^{2}-\sigma_{y}^{\infty} e_{y}^{0}\right\}+\max _{e_{\gamma}}\left\{w\left(e_{\gamma}, \boldsymbol{x}^{\prime}\right)\right.\right. \\
& \left.-w_{L}\left(e_{\gamma}, \boldsymbol{x}^{\prime}\right)\right\}+\tau_{\alpha}\left(\boldsymbol{x}^{\prime}\right) e_{\alpha}^{0}-\frac{1}{2} \tau_{\alpha}\left(\boldsymbol{x}^{\prime}\right)\left(\mu\left(\boldsymbol{x}^{\prime}\right)-\mu_{0}\right)^{-1} \tau_{\alpha}\left(\boldsymbol{x}^{\prime}\right) \\
& \left.-\frac{1}{2} \tau_{\alpha}\left(\boldsymbol{x}^{\prime}\right) \int_{V} d \mathbf{x} \Gamma_{\alpha \beta}\left(\boldsymbol{x}, \boldsymbol{x}^{\prime}\right) \tau_{\beta}(\boldsymbol{x})\right]
\end{aligned}
$$

The infimum here is attained when the parameters defining $\tau_{\alpha}$ satisfy (3.18) (or equivalently (3.39)), and then

$$
\begin{aligned}
\bar{U} \leq \frac{1}{V} \int_{V} d \boldsymbol{x}^{\prime} & {\left[\left\{\frac{1}{2} \mu_{0}\left[e_{y}^{0}\right]^{2}+\frac{1}{2} \tau_{\alpha}\left(\boldsymbol{x}^{\prime}\right) e_{y}^{0}-\sigma_{y}^{\infty} e_{y}^{0}\right\}+\max _{e_{\gamma}}\left\{w\left(e_{\gamma}, \boldsymbol{x}^{\prime}\right)\right.\right.} \\
& \left.\left.-w_{L}\left(e_{\gamma}, \boldsymbol{x}^{\prime}\right)\right\}\right] .
\end{aligned}
$$

It is known already, from study of the linear problem, that the coating and substrate do not interact in this Hashin-Shtrikman approximation. Consider first the integral over the region $V_{S}$ occupied by the substrate, in the limit as $L \rightarrow \infty$. There is only one phase, $r=M+1$, and so only one polarization which satisfies Equation (3.29).

It follows that

$$
\begin{aligned}
\frac{1}{V} \int_{V_{S}} d \boldsymbol{x}^{\prime}[ & \left\{\frac{1}{2} \mu_{0}\left[e_{y}^{0}\right]^{2}+\frac{1}{2} \tau_{\alpha}\left(\boldsymbol{x}^{\prime}\right) e_{y}^{0}-\sigma_{y}^{\infty} e_{y}^{0}\right\}+\max _{e_{\gamma}}\left\{w\left(e_{\gamma}, \boldsymbol{x}^{\prime}\right)\right. \\
& \left.\left.-w_{L}\left(e_{\gamma}, \boldsymbol{x}^{\prime}\right)\right\}\right] \\
= & \frac{H}{H+h}\left[-\frac{\left(\sigma_{y}^{\infty}\right)^{2}}{2 \mu_{M+1}}+\frac{(1-N)}{2(1+N)} e_{0} \sigma_{M+1}\left(\frac{\sigma_{M+1}}{e_{0} \mu_{M+1}}\right)^{\frac{1+N}{1-N}}\right]
\end{aligned}
$$

This expression is minimised with respect to $\mu_{M+1}$ when

$\mu_{M+1}=e_{0}\left(\sigma_{M+1}\right)^{\frac{1}{N}}\left(\sigma_{y}^{\infty}\right)^{\frac{N-1}{N}}$.

Substituted back into (5.11), this produces the result

$$
-\frac{N}{1+N} e_{0} \sigma_{M+1}\left(\frac{\sigma_{y}^{\infty}}{\sigma_{M+1}}\right)^{\frac{1+N}{N}}
$$

which is the exact complementary energy density in the substrate.

Consider now the contribution to $\bar{U}$ from the coating. It is convenient to define $\bar{U}_{C}^{H S}$ as the right side of (5.9), except that $V$ is replaced by $V_{C}$, that is, the intersection of $V$ with the coating. Evaluation of the infimum follows exactly as in the linear case and, in the limit as $L \rightarrow \infty$, reduces to

\footnotetext{
1 This calculation uses the long-established identity $\Gamma \mu_{0} \Gamma=\Gamma$ - see for instance Willis (1977).
}

$\bar{U}_{C}^{H S}=\frac{1}{2}\left(\mu_{0} e_{y}^{0}+\sum_{r=1}^{M} p_{r} \bar{\tau}_{y}^{(r)}\right) e_{y}^{0}+\sum_{r=1}^{M} p_{r} \frac{(1-N)}{2(1+N)} e_{0} \sigma_{r}\left(\frac{\sigma_{r}}{e_{0} \mu_{r}}\right)^{\frac{1+N}{1-N}}$

where

$\bar{\tau}_{y}^{(r)}=\frac{1}{h} \int_{-h}^{0} \tau_{y}^{(r)}(y) d y$

and $\tau_{y}^{(r)}(y)$ satisfy Equation (3.18). With this notation,

$\frac{1}{h} \int_{-h}^{0}\left\langle e_{y}\right\rangle d y=e_{y}^{0}-\sum_{r=1}^{M} p_{r} \bar{\tau}_{y}^{(r)} / \mu_{0}$

and it is elementary to check that (5.14) reduces to

$\bar{U}_{C}^{H S}=-\frac{1}{2 \bar{\mu}}\left(\sigma_{y}^{\infty}\right)^{2}+\sum_{r=1}^{M} p_{r} \frac{(1-N)}{2(1+N)} e_{0} \sigma_{r}\left(\frac{\sigma_{r}}{e_{0} \mu_{r}}\right)^{\frac{1+N}{1-N}}$,

where $\bar{\mu}$ is defined as in (4.3). Note that $\bar{U}_{L}^{H S} \equiv-\left(\sigma_{y}^{\infty}\right)^{2} /(2 \bar{\mu})$ represents the average over the coating of the potential energy associated with loading the comparison linear composite. Optimisation of (5.17) with respect to the parameters $\mu_{r}$ is further pursued in the case $M=2$.

\subsubsection{2-phase composite layer}

Further reduction is possible for the case of a two-phase layer, as follows. For the Hashin-Shtrikman upper bound, choose $\mu_{0}=\mu_{1}$ and write $s=\mu_{2} / \mu_{1}$. Then for the Hashin-Shtrikman upper bound, we require $0 \leq s \leq 1$. Since $\bar{\mu}$ is homogeneous of degree 1 in $\left(\mu_{1}, \mu_{2}\right)$, we can write

$\bar{\mu}=\mu_{1} g(s)$,

where the function $g(s)$ is known numerically from (3.2a). Choosing $\mu_{1}$ so as to minimise (5.17), with $s$ fixed, gives

$\mu_{1}=\left(e_{0}\right)^{-1}\left(\sigma_{y}^{\infty}\right)^{\frac{N-1}{N}}\left\{g(s)\left[p_{1} \sigma_{1}^{\frac{2}{1-N}}+p_{2} S^{\frac{N+1}{N-1}} \sigma_{2}^{\frac{2}{1-N}}\right]\right\}^{\frac{1-N}{2 N}}$

and then $\bar{U}_{C}^{H S}$ can be expressed as

$\bar{U}_{C}^{H S}=-\frac{N}{1+N} e_{0} \bar{\sigma}_{C}^{H S}\left(\frac{\sigma_{y}^{\infty}}{\bar{\sigma}_{C}^{H S}}\right)^{\frac{1+N}{N}}$

where

$\bar{\sigma}_{C}^{H S}=[g(s)]^{\frac{1+N}{2}}\left[p_{1} \sigma_{1}^{\frac{2}{1-N}}+p_{2} S^{\frac{N+1}{N-1}} \sigma_{2}^{\frac{2}{1-N}}\right]^{\frac{1-N}{2}}$.

The corresponding value of the average shear strain in the coating is

$\left\langle e_{y}\right\rangle=-\frac{\partial \bar{U}_{C}^{H S}}{\partial \sigma_{y}^{\infty}}=e_{0}\left(\frac{\sigma_{y}^{\infty}}{\bar{\sigma}_{C}^{H S}}\right)^{\frac{1}{N}}$.

Finally, the minimum of $\bar{U}_{C}^{H S}$ with respect to $s$, and the corresponding estimate for $\left\langle e_{y}\right\rangle$, are obtained by minimising $\bar{\sigma}_{C}^{H S}$ as given by Equation (5.21). This is done numerically using the MATLAB routine 'fminband': its algorithm is based on golden section search and parabolic interpolation. For the presentation of numerical results below, we shall label the minimum value of $\bar{\sigma}_{C}^{H S}$ as $\bar{\sigma}^{+}$. 


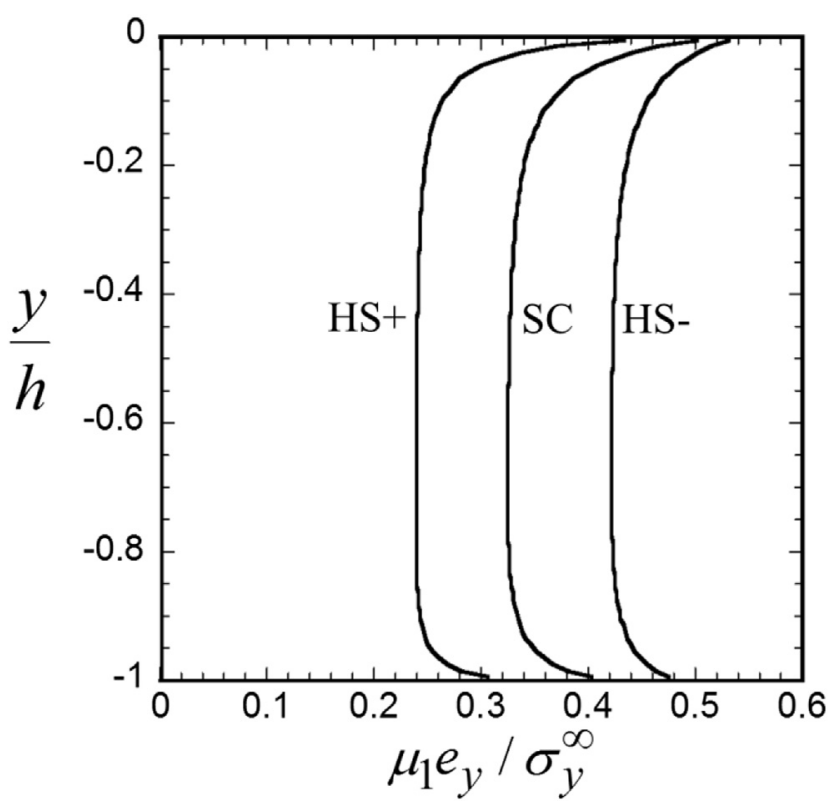

Fig. 2. Strain distribution within 2-phase composite coating for $\mu_{2} / \mu_{1}=10$, $p_{1}=p_{2}=0.5, \lambda / \mathrm{h}=0.1$. HS + denotes Hashin-Shtrikman upper bound, HS- denotes Hashin-Shtrikman lower bound and SC denotes self-consistent estimate.

\subsection{Lower estimate and self-consistent estimate}

A similar procedure to obtain strict Hashin-Shtrikman lower bounds for nonlinear composites is not available. Instead, estimates can be derived based on the linear Hashin-Shtrikman lower bounds and self-consistent estimates in the manner suggested by Ponte Castañeda and De Botton (1992). The idea is to evaluate (5.17), with $\bar{\mu}$ replaced by the Hashin-Shtrikman lower bound to produce a 'lower estimate' for $g(s)$ in (5.21). Now minimise $\bar{\sigma}_{C}^{H S}$ as given by Equation (5.21) with respect to $s$, and write the minimum value of $\bar{\sigma}_{C}^{H S}$ as $\bar{\sigma}^{-}$for later presentation of numerical results. Likewise, $\bar{\mu}$ in (5.17) is replaced by the Hashin-Shtrikman selfconsistent estimate $\mu_{S C}$ to obtain a self-consistent strength

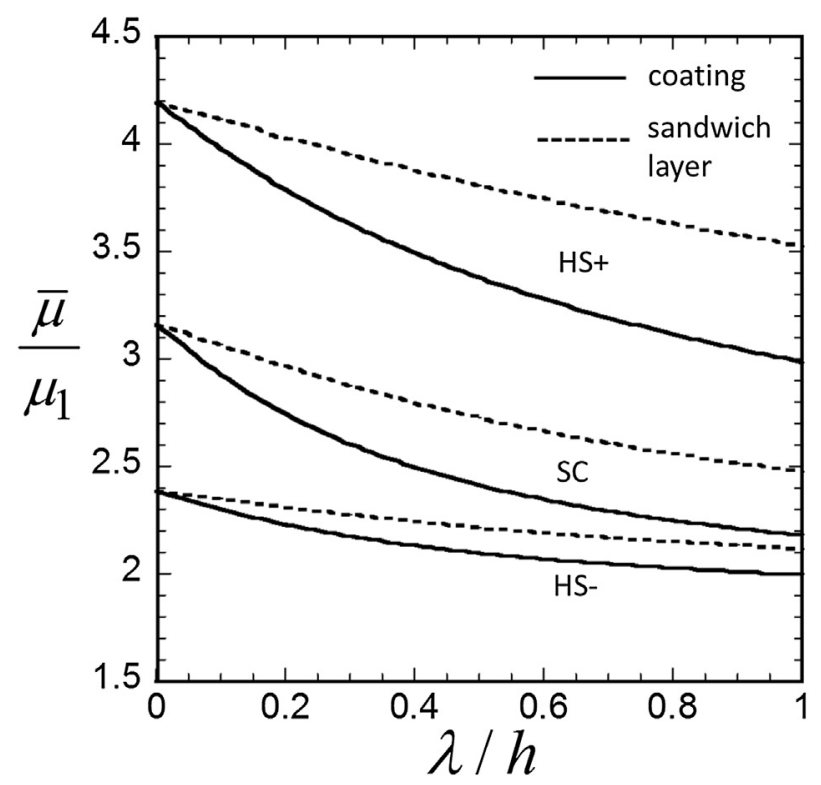

Fig. 3. Effective shear modulus of composite coating and sandwich layer for $\mu_{2} / \mu_{1}=10$ and $p_{1}=p_{2}=0.5$. HS + denotes Hashin-Shtrikman upper bound, HS- denotes Hashin-Shtrikman lower bound and SC denotes self-consistent estimate.
$\bar{\sigma}_{S C}=\bar{\sigma}_{C}^{H S}$ upon minimisation of (5.21) with respect to $s$.

\section{Results}

We begin by giving selected results for the Hashin-Shtrikman upper bound $\left(\mathrm{HS}^{+}\right)$, lower bound $\left(\mathrm{HS}^{-}\right)$and self-consistent approximation (SC) for the linear composite linear composite layer, both in the form of a coating and a sandwich layer. The nonlinear case is then reported for selected values of strain hardening exponent in the range $N=0$ to 0.3 .

\subsection{Linear composite}

Representative results for the distribution of shear strain within the surface coating are given in Fig. 2 for $\mu_{2} / \mu_{1}=10, p_{1}=p_{2}=0.5$, and the correlation length scale $\lambda$ equal to $h / 10$. Boundary layers of
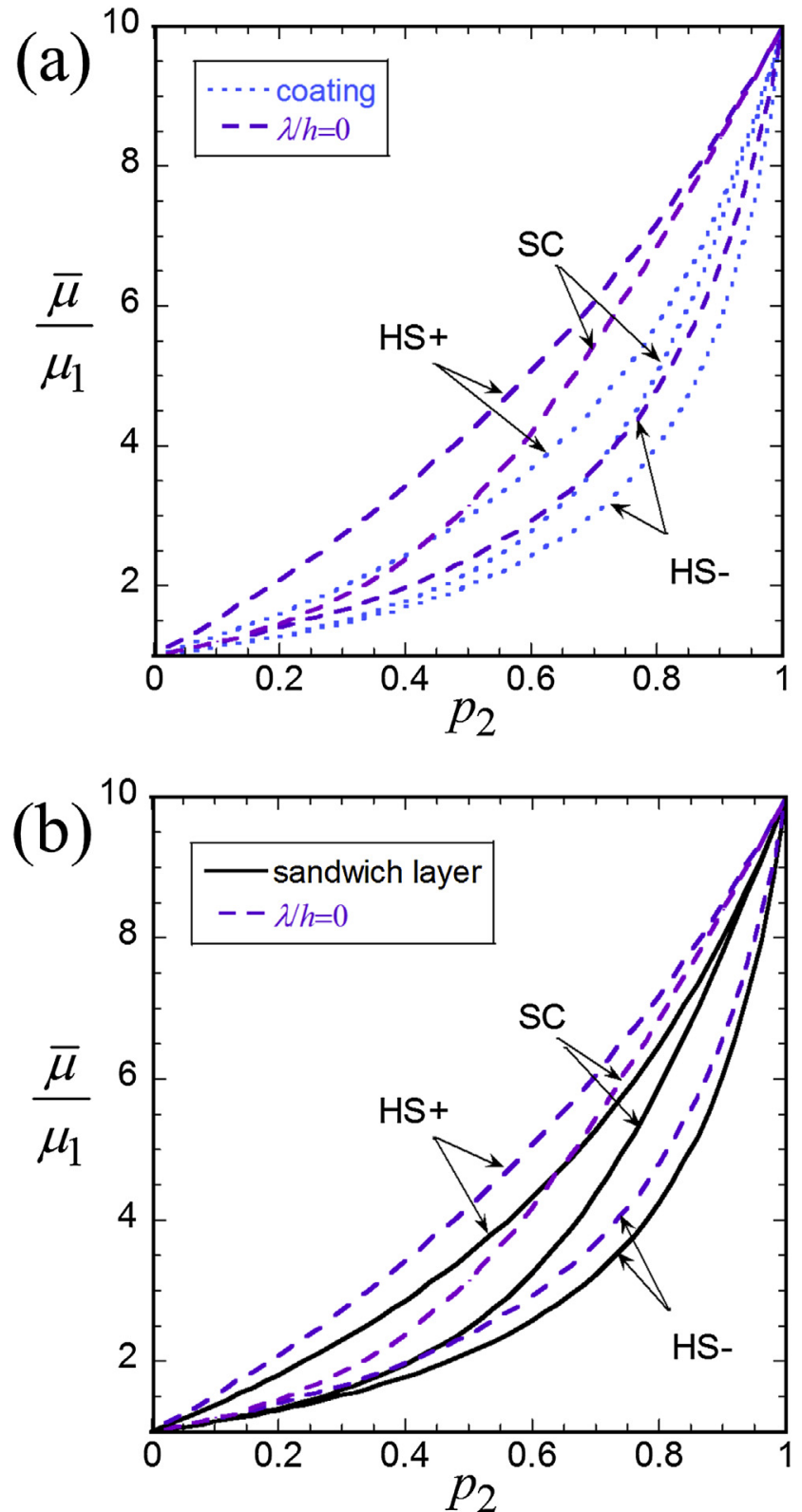

Fig. 4. Effective shear modulus of composite coating and sandwich layer for the choice $\lambda / h=0$, 1, with $\mu_{2} / \mu_{1}=10$. (a) coating and (b) sandwich layer. HS+ denotes Hashin-Shtrikman upper bound, HS- denotes Hashin-Shtrikman lower bound and SC denotes self-consistent estimate. 
thickness about $\lambda$ exist at the boundaries of the coating: the shear strain is amplified due to the presence of the free surface. In similar fashion, the ensemble-averaged value of shear strain is increased adjacent to the lower interface. This pattern is perhaps not surprising when the substrate is 'more compliant' than the coating. Conversely, if the substrate is 'less compliant' than the coating, the opposite trend might be expected. What must always be true is that the ensemble mean stress $\left\langle\sigma_{y}\right\rangle$ must equal $\sigma_{y}^{\infty}$, for any value of $y$, including $y=-h$. The strain profile, however, depends on the 'effective response' of the layer, which is sensitive to non-local interactions and so should depend on the modulus of the substrate. That it does not is a consequence of the Hashin-Shtrikman approximation. Since, however, this approximation is based on a variational structure (outlined explicitly in Section 5), it definitely provides bounds (and stationary estimates) for the (complementary) energy, as reflected in the 'effective stiffness' $\bar{\mu}$ of the layer as a
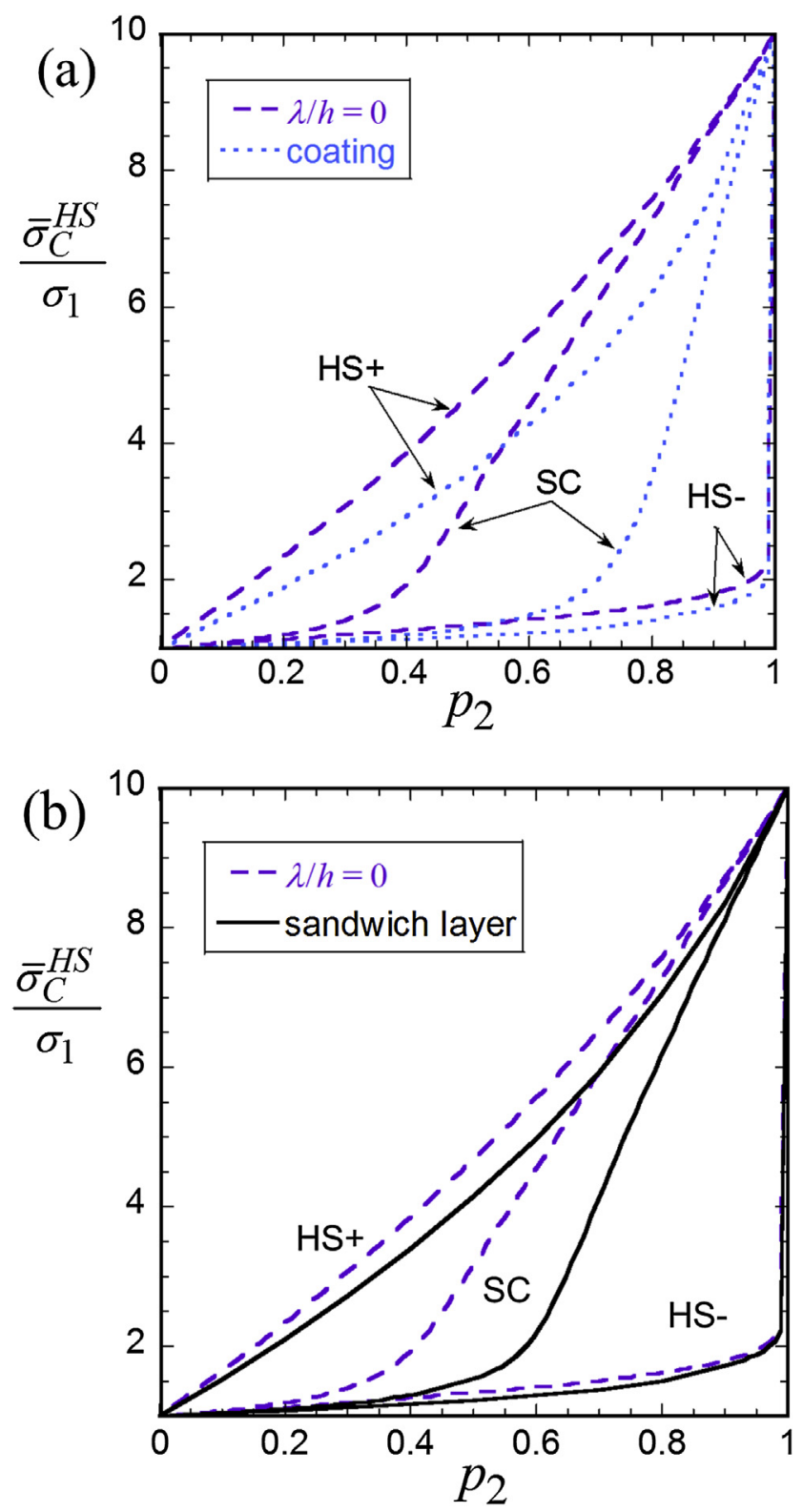

Fig. 5. Effective strength for $\mathrm{N}=0.1, \sigma_{2} / \sigma_{1}=10$ and $\lambda / h=0,1$ for (a) coating and (b) sandwich layer. HS + denotes Hashin-Shtrikman upper bound, HS- denotes Hashin-Shtrikman lower estimate and SC denotes self-consistent estimate. whole. More detailed exploration of the effect of the substrate on the effective response would require either allowance for higherorder statistics or direct numerical simulation.

Predictions for the averaged shear stiffness of the coating and sandwich layer, normalized by $\mu_{1}$, are plotted as a function of $\lambda / h$ in Fig. 3 , for $p_{2}=0.5$ and $\mu_{2} / \mu_{1}=10$. Upper bounds, lower bounds and self-consistent estimates are included. All values tend to the corresponding Hashin-Shtrikman estimates for the effective modulus of the bulk composite as $\lambda / h \rightarrow 0$. For both coating and sandwich layer, $\bar{\mu} / \mu_{1}$ decreases with increasing ratio of $\lambda / h$ : the boundary layers increase the local compliance and lead to a drop in overall macroscopic stiffness of layer. The wide separation of the bounds leaves uncertainty about the exact value, but all our estimates display the same trend. Our 'recommended' estimate is the selfconsistent (SC). We note in passing that for the choice $p_{2}=0.5$ the self-consistent bulk-value (2.8) reduces to $\mu_{\mathrm{SC}}=\sqrt{\mu_{1} \mu_{2}}$, which is exact for a material whose phases are distributed symmetrically and so can be interchanged, see for example Milton (2002).

The sensitivity of macroscopic stiffness of the composite layer (averaged over the thickness) is explored in Fig. 4, for the extreme cases of $\lambda / h=0,1$, with $\mu_{2} / \mu_{1}=10$ and for all values of volume fraction $p_{2}$ of phase 2 . Results for the coating are given in Fig. 4a and for the sandwich layer in Fig. 4b. We emphasise that the choice $\lambda /$ $h=0$ is the conventional formulation for a bulk 2-phase composite (boundary layers of vanishing thickness), and is the same for both coating and sandwich layer. For the choice $\lambda / h=1$, the layer stiffness (whether coating or sandwich layer) is below that of the conventional bulk composite $(h=\infty)$ but increases with increasing $p_{2}$ in a similar manner. Note that the self-consistent estimate is close to $\mathrm{HS}^{+}$at $p_{2}$ close to zero, and lies close to $\mathrm{HS}^{-}$at $p_{2}$ close to unity, for both $\lambda / h=0$ and 1 .

\subsection{Non-linear composite}

Estimates $\bar{\sigma}_{C}^{H S}$ of the effective flow strength of the coating and sandwich layer are determined by minimization of (5.21) with respect to the ratio $s$ of moduli in the linear comparison medium, as detailed in Section 5, for the case of an upper bound $\bar{\sigma}^{+}$, lower

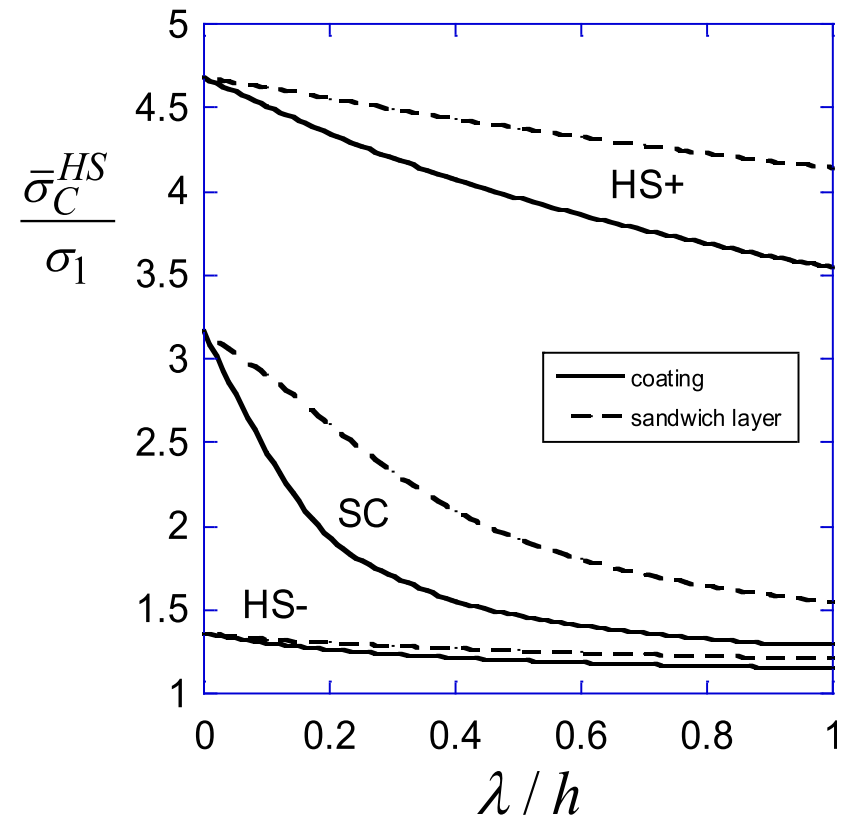

Fig. 6. Effective strength of composite coating and sandwich layer for $N=0.1, \sigma_{2} /$ $\sigma_{1}=10$ and $p_{2}=0.5$. HS + denotes Hashin-Shtrikman upper bound, HS- denotes Hashin-Shtrikman lower estimate and SC denotes self-consistent estimate. 

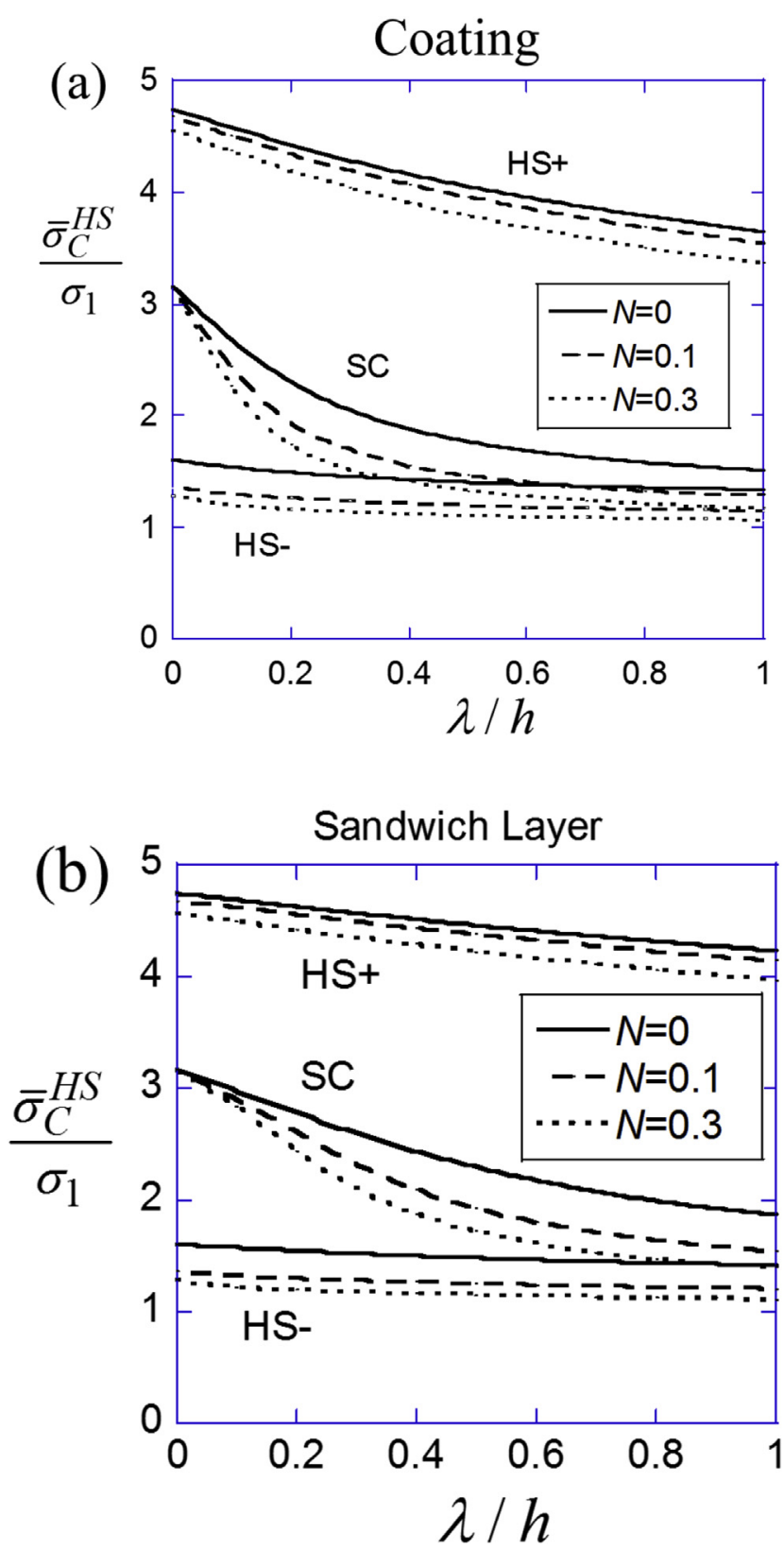

Fig. 7. Effective strength for $\sigma_{2} / \sigma_{1}=10, p_{2}=0.5$ and selected values of $N$ for (a) coating and (b) sandwich layer. HS+ denotes Hashin-Shtrikman upper bound, HS- denotes Hashin-Shtrikman lower estimate and SC denotes self-consistent estimate.

estimate $\bar{\sigma}^{-}$and self-consistent estimate $\bar{\sigma}_{C}^{H S}$. Results are limited to the choice $\sigma_{2} / \sigma_{1}=10$ and $N=0.1$ in Figs. 5 and $6: \bar{\sigma}_{C}^{H S} / \sigma_{1}$ is plotted as a function of $p_{2}$ (with $\lambda / h=0,1$ ) in Fig. 5 whereas $\bar{\sigma}_{C}^{H S} / \sigma_{1}$ is plotted as a function of $\lambda / h$ (with $p_{2}=0.5$ ) in Fig. 6. The plot of $\bar{\sigma}_{C}^{H S} / \sigma_{1}$ versus $p_{2}$ in Fig. 5 is analogous to that of $\bar{\mu} / \mu_{1}$ versus $p_{2}$ in Fig. 4 , for the linear case, with similar trends. It is clear from Fig. 5 that the upper bound and lower estimate are more widely spaced for the strongly non-linear case $(N=0.1)$ than for the linear case of Fig. 4 . There is a moderate drop in both upper bound strength and the self-consistent strength when $\lambda / h$ is increased from zero to unity, due to the emergence of soft boundary layers within the coating (Fig. 5a) and sandwich layer (Fig. 5b). And, as $p_{2}$ is increased from zero to unity, the self-consistent estimate for $\bar{\sigma}_{C}^{H S}$ increases from the lower estimate to the upper bound.

The sensitivity of effective flow strength of the coating and sandwich layer to length scale $\lambda / h$ is shown in Fig. 6. As for the linear case (recall Fig. 3), the strength drops with increasing $\lambda / h$ for all bounds and estimates, and the drop in strength is greater for the coating than for the sandwich layer.

Finally, it is instructive to plot $\bar{\sigma}_{C}^{H S} / \sigma_{1}$ as a function of $\lambda / h$ for selected values of strain hardening exponent $N$, see Fig. 7 a for the coating and Fig. $7 \mathrm{~b}$ for the sandwich layer. In both plots we limit attention to $\sigma_{2} / \sigma_{1}=10$ and $p_{2}=0.5$. We note only a mild effect of $N$ upon the strength over the practical range $0 \leq N \leq 0.3$. Further, we note that the value $\bar{\sigma}_{C}^{H S} / \sigma_{1}$ for the self-consistent estimate is independent of $N$ at $\lambda / h=0$. Direct analytical minimisation of $\bar{\sigma}_{C}^{H S}$ in (5.21) is possible for the case $p_{2}=0.5$, with the result $\bar{\sigma}_{C}^{H S}=\sqrt{\sigma_{1} \sigma_{2}}$, which closely parallels the result for the linear composite $\bar{\mu}_{S C}=\sqrt{\mu_{1} \mu_{2}}$.

\section{Concluding remarks}

A Hashin-Shtrikman variational approach is developed for composite layers, with strict upper and lower bounds for the linear solid, and bounds and estimates for the non-linear case. Our study reveals the existence of boundary layers of increased compliance at interfaces, including the free surface. For the non-linear case, the effective strength of the layer is only mildly sensitive to the choice of strain hardening exponent. The analytical development shows explicitly that the linear (and non-linear) effective responses of a layer are independent of the choice of substrate modulus. Further work is needed to explore the accuracy of this somewhat surprising result by performing explicit finite element simulations or by assuming 3 point statistics (rather than 2-point statistics as assumed here).

\section{Acknowledgements}

NAF is grateful for financial support in the form of an ERC MULTILAT grant 669764, and to the US ONR (N62909-14-1-N232, project manager, Dr. Dave Shifler).

\section{References}

Drugan, W.J., 2003. Two exact micromechanics-based nonlocal constitutive equations for random linear elastic composite materials. J. Mech. Phys. Solids 51, 1745-1772.

Hill, R., 1964. Theory of mechanical properties of fibre-strengthened materials. I. Elastic behaviour. J. Mech. Phys. Solids 12, 199-212.

Hill, R., 1965. A self-consistent mechanics of composite materials. J. Mech. Phys. Solids $13,213-222$.

Milton, G.W., 2002. The Theory of Composites. Cambridge University Press.

Ponte Castañeda, P., 1991. The effective mechanical properties of nonlinear isotropic composites. J. Mech. Phys. Solids 39, 45-71.

Ponte Castañeda, P., 1992. New variational principles in plasticity and their application to composite materials. J. Mech. Phys. Solids 40, 1757-1788.

Ponte Castañeda, P., De Botton, G., 1992. On the homogenized yield strength of twophase composites. Proc. Roy. Soc. A438, 419-431.

Smyshlyaev, V.P., Fleck, N.A., 1995. Bounds and estimates for the overall plastic behaviour of composites with strain gradient effects. Proc. Roy. Soc. A451, 795-810.

Walpole, L.J., 1969. On the overall elastic moduli of composite materials. J. Mech. Phys. Solids 17, 235-251.

Willis, J.R., 1977. Bounds and self-consistent estimates for the overall moduli of anisotropic composites. J. Mech. Phys. Solids 25, 182-202. 